\title{
Compact brain MRI
}

\author{
A prototype device for performing magnetic-resonance-imaging scans of the brain that integrates a lightweight \\ permanent magnet and low-power gradient coils is suitable for eventual use at the bedside.
}

\section{J. Michael Tyszka}

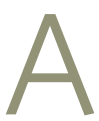

typical system for magnetic resonance imaging (MRI) weighs more than 4 metric tons, and requires magnetic and radiofrequency shielding, substantial electrical power and other specialized infrastructure ${ }^{1}$. Although commonly available in most large urban hospitals and in outpatient imaging facilities in developed countries, access to diagnostic MRI can be considerably limited in rural areas and practically non-existent in many developing countries (there are about 7 MRI scanners per million people in the United Kingdom and 50 units per million people in Japan ${ }^{2}$, but less than 0.5 units per million people in countries from sub-Saharan West Africa ${ }^{3}$ ). Access to MRI scanners could be improved with low-cost MRI devices with a small footprint that could be used at the point of care in underserved communities and in high-income settings alike. This has long motivated academic and industrial researchers working in medical imaging.

Demand for MRI scanners with increased sensitivity and signal-to-noise ratio for clinical and research uses has led to devices with ever-increasing magnetic fields. High-field MRI systems involve a substantial investment and financial burden, also because of the need for a specialized shielded room with structural support, seismic isolation and ongoing service and maintenance. In fact, in many developed countries, the fraction of the healthcare budget that supports non-invasive MRI and computed tomography imaging is substantial ${ }^{2}$. Yet the drive towards higher-field imaging systems does not imply that there aren't benefits in developing smaller and lower-field MRI scanners. In fact, two low-field head-only MRI systems, approved by the United States Food and Drug Administration in 2020, indicate continued industrial commitment to this class of scanner. The cryogen-free 0.5-T device from Synaptive Medical requires only $25 \mathrm{~m}^{2}$ of floorspace, and is designed for use at small clinics and emergency departments in hospitals; however, it is not a bedside scanner. The 200-kg, 64-mT cart-mounted
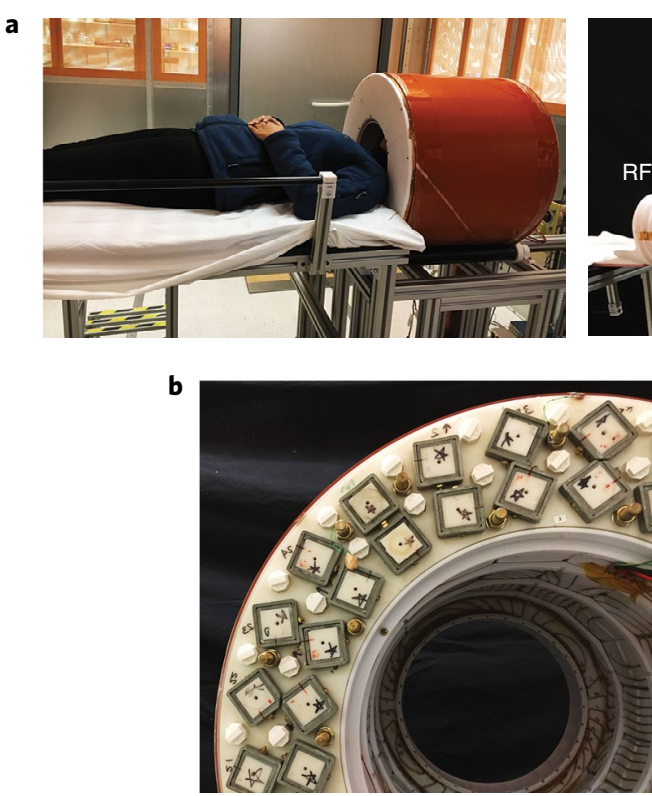
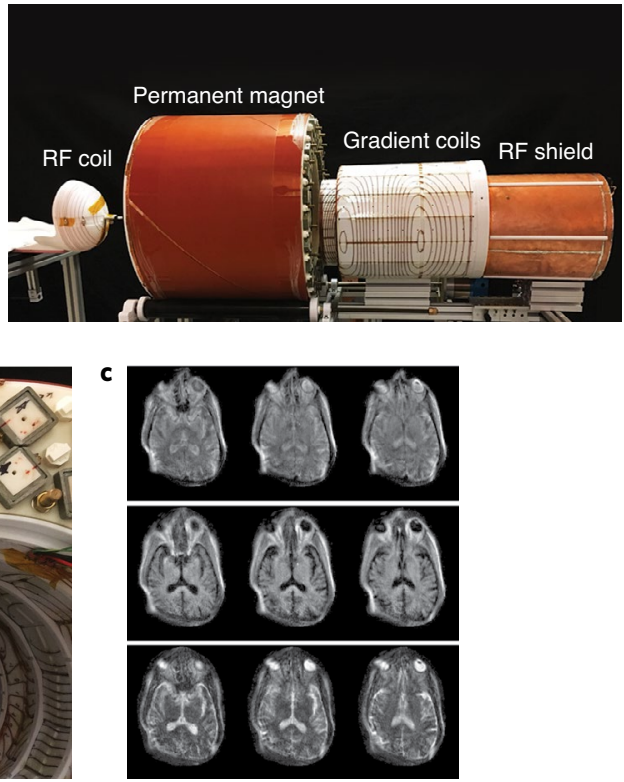

Fig. 1 | A prototype of a portable MRI scanner for the head. $\mathbf{a}$, A permanent rare-earth magnet, weighing slightly over $120 \mathrm{~kg}$ when including its associated gradient coils and radiofrequency (RF) coils, fitted around the head of a volunteer (left). The entire MRI system (right), which includes amplifiers, console and carts, weighs approximately $230 \mathrm{~kg}$ and draws about $800 \mathrm{~W}$ of electrical power when scanning. $\mathbf{b}$, The static polarizing and gradient magnetic fields are generated by an optimized Halbach array of permanent magnets of stock neodymium, which saves weight, power consumption and cost, and minimizes stray magnetic fields. c, Transverse slices of the imaged brain of a volunteer, exemplifying three clinically relevant image contrasts (from top to bottom: proton density, T1-weighted and T2-weighted; images in each row are from three different axial slice locations at the approximate level of the eyes). Figure reproduced with permission from ref. ${ }^{4}$, Springer Nature Ltd.

MRI system from Hyperfine can perform neuroimaging at the bedside, has low power requirements, and is the first commercial ultra-small MRI system. Clarissa Cooley and colleagues now report in Nature Biomedical Engineering the development and testing, in volunteers, of a small and lightweight prototype MRI scanner tailored for imaging the head ${ }^{4}$ (Fig. 1a).

Similar to the scanner from Hyperfine, Cooley and co-authors' prototype device is portable, and needs minimal power and housing requirements. Yet differently from the commercial scanner, the device uses an array of nearly 900 permanent neodymium magnets arranged in a nearly cylindrical
Halbach configuration, which generates a magnetic flux density of approximately $80 \mathrm{mT}$ (Fig. 1b). The Halbach array is a popular choice for one-sided and compact cylindrical magnets; it generates a relatively homogeneous magnetic field within the bore of the cylinder, and minimal stray flux outside the array $^{5-7}$. The authors used mass-produced rare-earth magnets, which are inexpensive compared with the monolithic permanent magnets (sometimes custom-made) used in clinical MRI scanners. The array of magnets is asymmetric, and incorporates passive shimming elements to refine the static magnetic field within the bore. 
MRI relies on the spatial encoding of the resonance frequency of nuclear spins. This is achieved via highly linear magnetic-field gradients in one or more directions. Yet distinct from traditional MRI magnets, Cooley and colleagues designed an array of magnets that generates a constant and approximately linear magnetic-field gradient vertically. This replaces one of the three gradient axes used for the frequency encoding of spatial position, which is conventionally done by passing current through cylindrically wound coils within the bore of the magnet. Although it can no longer be pulsed, this constant gradient does not need to draw additional power, and thus eliminates one of three heavy gradient amplifiers that a typical MRI system uses. The remaining two gradient axes employ conventional optimized coil windings on cylindrical formers. An optimized spiral-wound 'cap' coil supports radiofrequency excitation and reception of the MRI signal from the participant's head ${ }^{8}$.

The use of a constant (rather than switching) readout magnetic-field gradient requires the use of swept frequency or 'chirp' pulses to accommodate the broad range of resonance frequencies that result from the constant readout gradient. A constant-field gradient also prevents the use of gradient echo-signal refocusing (which speeds up imaging) and thus limits pulse sequences to spin-echo designs, such as the modified multiple echo rapid acquisition and relaxation enhancement (RARE) pulse sequence. Although multiple spin-echo sequences generally cause more tissue heating than gradient-echo methods at high fields, this is more than compensated by the low polarization field of the prototype device, and would allow a relatively efficient signal acquisition in a clinically practical timeframe. Ultimately, whole-brain images (Fig. 1c) can be generated in $8-12 \mathrm{~min}$ at a spatial volume resolution of approximately $20 \mu \mathrm{l}$ $(1.3 \mathrm{~mm} \times 2.2 \mathrm{~mm} \times 6.8 \mathrm{~mm})$. The basic RARE sequence can be pre-weighted with clinically relevant image contrasts ( $\mathrm{T} 1$ and $\mathrm{T} 2$, in particular), and future adaptations are expected to enable diffusion-weighting.
The nonlinearity of the constant-field gradient requires a more complex image-reconstruction process involving the acquisition of calibration-field maps and a generalized iterative process?

Naturally, the miniaturization of MRI scanners involves compromises in image quality. The low polarizing field implies a correspondingly reduced sensitivity, which forces longer scan times and leads to lower spatial resolution than achieved in typical high-field clinical scanners. External electromagnetic interference is a perennial concern for portable systems, and in the absence of a Faraday-shielded room remains an outstanding challenge despite the use of radiofrequency shielding around the magnet array. Active interference detection and compensation during image reconstruction (currently under development by Cooley and co-authors) may mitigate external electromagnetic interference. And despite the thorough optimization of the magnet array, passive shimming and the use of a generalized image-reconstruction process accounting for residual nonlinearity of the readout gradient, images from the prototype device show residual geometric distortion, particularly at the surface of the head (Fig. 1c). Such artefacts can be readily addressed via refinement of the magnet array and via iterative reconstruction approaches, and are likely to improve in subsequent iterations of the prototype.

Because it dispenses from a superconducting magnet, Cooley and co-authors' portable scanner does not require cryogenic refrigeration or access to liquid helium (which come with large service and maintenance costs). Optimization of heavy electrical components such as the gradient power amplifiers could reduce the total weight of the system to around $160 \mathrm{~kg}$ (comparable to a cart-mounted clinical ultrasound system), and thus allow a single operator to move the system on a cart to the bedside. The power consumption of the authors' prototype device is sufficient for it to be supported by a solar battery system or a diesel generator with appropriate conditioning. This could allow broader uses of the device.
In diagnostic imaging of the brain, the advantages of MRI over ultrasound and digital X-ray imaging are well established. Conventional cranial ultrasound generally requires an acoustic window through the skull to perform optimally, and consequently is used widely for clinical neurovascular imaging in neonates while the fontanelle is open. Digital X-rays have limited soft-tissue contrast and deliver ionizing radiation. MRI is not limited by the availability of acoustic windows, employs non-ionizing radiofrequency radiation, and offers superior soft-tissue contrast and essentially unlimited tissue penetration. It is particularly valuable in the assessment of acute stroke and of increases in intracranial pressure. Therefore, a portable MRI scanner for the head would be most valuable in emergency care and critical care. In view of the two recent regulatory clearances of low-field, small-footprint MRI head scanners, Cooley and co-authors' prototype may eventually also offer wider access to magnetic-resonance neuroimaging to underserved communities.

\section{J. Michael Tyszka (D) $ه$ \\ Caltech Brain Imaging Center, California Institute of Technology, Pasadena, CA, USA. \\ $凶_{e-m a i l: j m t @ c a l t e c h . e d u}$}

Published online: 16 March 2021

https://doi.org/10.1038/s41551-021-00702-3

References

1. Heye, T. et al. Radiology 295, 593-605 (2020).

2. Papanicolas, I., Woskie, L. R. \& Jha, A. K. JAMA 319, 1024-1039 (2018).

3. Ogbole, G. I., Adeyomoye, A. O., Badu-Peprah, A., Mensah, Y. \& Nzeh, D. A. Pan Afr. Med. J. 30, 240 (2018).

4. Cooley, C. Z. et al. Nat. Biomed. Eng. https://doi.org/10.1038/ s41551-020-00641-5 (2020).

5. Chang, W.-H., Chen, J.-H. \& Hwang, L.-P. Magn. Reson. Imaging 24, 1095-1102 (2006).

6. Manz, B., Benecke, M. \& Volke, F. J. Magn. Reson. 192, 131-138 (2008)

7. Tewari, S., O'Reilly, T. \& Webb, A. J. Magn. Reson. 324, 106923 (2021).

8. LaPierre, C. D., Sarracanie, M., Waddington, D. E. J. \& Rosen, M. S. A single channel spiral volume coil for in vivo imaging of the whole human brain at $6.5 \mathrm{mT}$. In Proc. 23rd Annual Meeting of the International Society for Magnetic Resonance in Medicine (ISMRM) Abstract no. 1793 (ISMRM, 2015).

9. Lin, F.-H. et al. Magn. Reson. Med. 68, 1145-1156 (2012).

Competing interests

The author declares no competing interests. 